\title{
Vlasov-Maxwell Simulations in Singular Geometries
}

\author{
Franck Assous ${ }^{1}$ and Patrick Ciarlet Jr. ${ }^{2}$ \\ 1 Bar-Ilan University, 52900 Ramat-Gan, Israel \\ and College of Judea\&Samaria, Ariel, Israel \\ franckassous@netscape.net \\ 2 ENSTA,32 bvd Victor, 75739, Paris Cedex 15 \\ ciarlet@ensta.fr
}

\begin{abstract}
This paper is devoted to the solution of the time-dependent Vlasov-Maxwell equations in singular geometries, i.e. when the boundary includes reentrant corners or edges. Indeed, computing the electromagnetic fields in this case is a challenge per se, as these geometrical singularities generate very strong solutions in their neighborhood. Moreover, they have also an influence over the solution of the Vlasov equation, through the coupling. We propose here a method to solve this problem, illustrated by numerical examples.
\end{abstract}

\section{Introduction}

The numerical simulation of charged particles beams or plasma physics phenomena requires methods of solution for the time-dependent coupled Vlasov-Maxwell system. Within this framework, we developed a numerical method (see 1]), with continuous approximations of the electromagnetic field, which is recommended in order to reduce spurious oscillations. In addition, the time-stepping numerical scheme, which is explicit by construction, can be solved very efficiently. Finally, the conditions on the divergence of the fields are considered as constraints, and are dualized, using a Lagrange multiplier, which yields a saddle-point variational formulation.

In practical examples, the boundary of the computational domain includes reentrant corners and/or edges (called geometrical singularities) that generate strong fields. Hence, they require a careful computation of the electromagnetic field in their neighborhood.

We developed a method (see [2]), the so-called Singular Complement Method (SCM hereafter), which consists in splitting the space of solutions into a twoterm sum. The first subspace is made of regular fields, and coincides with the whole space of solutions, provided that the domain is either convex or regular. So, one can compute the regular part of the solution with the help of an ad hoc - classical - method [1]. The second one is called the subspace of singular fields, and is computed with the help of specifically designed methods: they originate from relations between the electromagnetic singularities and the singularities of the Laplace operator. 
As a first attempt, the Singular Complement Method was constructed in a divergence-free framework (cf. [2]). When the divergence of the electric field no longer vanishes, $\operatorname{div} \mathcal{E}=f(t)$, with $f \neq 0$, a simple solution is to substract a gradient, to reach the divergence-free field

$$
\tilde{\mathcal{E}}=\mathcal{E}-\operatorname{grad} \phi .
$$

Unfortunately, to determine $\phi$, one has to solve the time-dependent (via the data) problem

$$
-\Delta \phi=f(t),
$$

which slows down drastically the numerical implementation.

To alleviate this drawback, we studied in detail different splittings of the electromagnetic space, which could be used for the SCM (cf. [3]). In addition to the divergence-free splittings, we propose new splittings, direct and possibly orthogonal, with curl-free singular fields, or with singular fields with $L^{2}$ divergence, etc.

As an important application - actually, the origin of this study - we present the computation of strong electromagnetic fields, via the numerical solution to the coupled, non linear, Vlasov-Maxwell system of equations. In Section 2, we recall Vlasov-Maxwell equations, and describe the $S C M$ well-suited to this framework. Section 3 is devoted to the numerical algorithms. Numerical experiments of the coupled Vlasov-Maxwell are presented in Section 4

\section{Vlasov-Maxwell Equations}

Let $\Omega$ be a bounded, open, polyhedral subset of $\mathbb{R}^{3}$, and $\Gamma$ its boundary. We denote by $\mathbf{n}$ the unit outward normal to $\Gamma$. The Vlasov equation models the transport of charged particles, under the influence of an electromagnetic field. The Vlasov equation reads

$$
\frac{\partial f}{\partial t}+\mathbf{v} \cdot \nabla_{\mathbf{x}} f+\frac{\mathbf{F}}{m} \cdot \nabla_{\mathbf{v}} f=0 .
$$

Above, the unknown $f$ is the distribution function of the particles, $f(\mathbf{x}, \mathbf{v}, t), \mathbf{v}$ stands for the velocities of the particles, $m$ is the mass of a particle, and

$$
\mathbf{F}=e(\mathcal{E}+\mathbf{v} \times \mathcal{B})
$$

is the the well-known Lorentz force. The electric and magnetic fields $\mathcal{E}$ and $\mathcal{B}$ are solution of the Maxwell equations in vacuum

$$
\begin{aligned}
& \frac{\partial \mathcal{E}}{\partial t}-c^{2} \operatorname{curl} \mathcal{B}=-\frac{1}{\varepsilon_{0}} \mathcal{J}, \\
& \frac{\partial \mathcal{B}}{\partial t}+\operatorname{curl} \mathcal{E}=0, \\
& \operatorname{div} \mathcal{E}=\frac{\rho}{\varepsilon_{0}} \\
& \operatorname{div} \mathcal{B}=0
\end{aligned}
$$


where $c, \varepsilon_{0}$ and $\mu_{0}$ are respectively the light velocity, the electric and magnetic constants $\left(\varepsilon_{0} \mu_{0} c^{2}=1\right)$. The charge and current densities $\rho$ and $\mathcal{J}$ have to verify the charge conservation equation

$$
\frac{\partial \rho}{\partial t}+\operatorname{div} \mathcal{J}=0
$$

Let us assume for simplicity that the boundary $\Gamma$ is a perfect conductor, so we have $\mathcal{E} \times \mathbf{n}=0$ and $\mathcal{B} \cdot \mathbf{n}=0$ on $\Gamma$. These equations are supplemented with appropriate initial conditions. Remark that the coupling occurs on the one hand, by the right-hand sides of Maxwell equations, $\rho$ and $\mathcal{J}$, which are computed from the solution to the Vlasov equation $f(\mathbf{x}, \mathbf{v}, t)$, thanks to the relations

$$
\rho=\int_{\mathbf{v}} f d \mathbf{v}, \quad \mathcal{J}=\int_{\mathbf{v}} f \mathbf{v} d \mathbf{v} ;
$$

on the other hand, the electromagnetic field $(\mathcal{E}, \mathcal{B})$ determines the forces that act on the particles via the Lorentz force $\mathbf{F}$.

Geometrical singularities have no effect per se on the regularity of the solution to the Vlasov equation. Therefore, we resort to a particle method [4]: it consists in approximating the distribution function $f(\mathbf{x}, \mathbf{v}, t)$ by a linear combination of Dirac masses in the phase space $(\mathbf{x}, \mathbf{v})$

$$
f(\mathbf{x}, \mathbf{v}, t) \simeq \sum_{k} w_{k} \delta\left(\mathbf{x}-\mathbf{x}_{k}(t)\right) \delta\left(\mathbf{v}-\mathbf{v}_{k}(t)\right),
$$

where each term of the sum can be identified with a macro-particle, characterized by its weight $w_{k}$, its position $\mathbf{x}_{k}$ and its velocity $\mathbf{v}_{k}$. This distribution function is a solution of the Vlasov equation (1) if and only if $\left(\mathbf{x}_{k}, \mathbf{v}_{k}\right)$ is a solution of the differential system:

$$
\begin{aligned}
\frac{d \mathbf{x}_{k}}{d t} & =\mathbf{v}_{k} \\
\frac{d \mathbf{v}_{k}}{d t} & =\mathbf{F}\left(\mathbf{x}_{k}, \mathbf{v}_{k}\right),
\end{aligned}
$$

which describes the time evolution of a macro-particle $k$, submitted to the electromagnetic force $\mathbf{F}$.

The system (10,11) can be numerically solved by an explicit time discretization algorithm. We used a leapfrog scheme which is well-adapted in this case. Given a constant time step $\Delta t$, the particles positions are defined at time $t_{n}=$ $n \Delta t$ and the particle velocities are computed at time $t_{n+1 / 2}=(n+1 / 2) \Delta t$. We refer the reader to [5] for more details. It is a classical approach in Particle In Cell (PIC) approach.

Even if geometrical singularities have no effect on the regularity of the solution to the Vlasov equation, they have an influence over $f$, through the coupling, i.e through the force $\mathbf{F}$. Hence, the electromagnetic field must be computed accurately. To this purpose, the $S C M$ was introduced in [2, first for divergencefree problems. 
Let us present here a way to generalize this approach to Vlasov-Maxwell problems. As we are interested in non divergence-free solutions, we will only present the electric formulation. Details on the magnetic counterpart can be found in [6]. Let us recall the definitions of the following spaces

$$
\begin{aligned}
\mathbf{H}(\operatorname{curl}, \Omega) & =\left\{\mathbf{u} \in \mathbf{L}^{2}(\Omega), \operatorname{curl} \mathbf{u} \in \mathbf{L}^{2}(\Omega)\right\} \\
\mathbf{H}(\operatorname{div}, \Omega) & =\left\{\mathbf{u} \in \mathbf{L}^{2}(\Omega), \operatorname{div} \mathbf{u} \in L^{2}(\Omega)\right\} \\
\mathbf{H}^{1}(\Omega) & =\left\{\mathbf{u} \in \mathbf{L}^{2}(\Omega), \operatorname{grad} \mathbf{u} \in \mathbf{L}^{2}(\Omega)\right\} .
\end{aligned}
$$

We define the space of electric fields $\mathcal{E}$, called $\mathbf{X}$,

$$
\mathbf{X}=\left\{\mathbf{x} \in \mathbf{H}(\mathbf{c u r l}, \Omega) \cap \mathbf{H}(\operatorname{div}, \Omega): \mathbf{x} \times \mathbf{n}_{\mid \Gamma}=0\right\} .
$$

When the domain is convex, the spaces $\mathbf{X}$ is regular. That is not the case anymore in a singular domain (see for instance [7]). Hence, one introduces the regular subspace for electric fields (indexed with $R$ )

$$
\mathbf{X}_{R}=\mathbf{X} \cap \mathbf{H}^{1}(\Omega),
$$

which is actually closed in $\mathbf{X}$ (cf. [6]), so that one is able to consider its orthogonal, and then define a two-part, direct, and orthogonal sum of the space. The orthogonal subspace is called singular subspacs (indexed with ${ }_{S}$ ). One can write

$$
\mathbf{X}=\mathbf{X}_{R} \stackrel{\perp \mathbf{x}}{\oplus} \mathbf{X}_{S}
$$

Thus, one can split an element $\mathbf{u}$ of the space $\mathbf{X}$ into an orthogonal sum of a regular part and of a singular part: $\mathbf{u}=\mathbf{u}_{R}+\mathbf{u}_{S}$. We have now to characterize the singular electric fields. Following [6], elements $\mathbf{x}_{S} \in \mathbf{X}_{S}$ satisfy

$$
\begin{aligned}
& \Delta \mathbf{x}_{S}=0 \quad \text { in } \Omega, \\
& \mathbf{x}_{S} \times \mathbf{n}_{\mid \Gamma}=0 .
\end{aligned}
$$

\section{Numerical Algorithms}

The numerical method consists in first computing numerically the basis of the singular subspace. Then we solve the problem by coupling a classical method (to compute the regular part of the solution) to the linear system, which allows to compute the singular part of the solution.

To compute $\mathbf{x}_{S} \in \mathbf{X}_{S}$, we introduce its divergence- and curl-free parts $\mathbf{v}_{S}$ and $\mathbf{l}_{S}$, which verify the following Helmholtz decomposition

$$
\mathbf{x}_{S}=\mathbf{v}_{S}+\mathbf{l}_{S}
$$

We also introduce $s_{N}$ and $s_{P}$, the non-vanishing (singular) solutions of

$$
\Delta s_{N}=0, \quad \Delta s_{P}=0 \quad \text { in } \Omega,
$$


respectively with Neumann and Dirichlet homogeneous boundary condition. Assume that $s_{N}$ and $s_{P}$ are known, then one can use the singular mappings (see [6]), to find $\phi_{S}$ and $\psi_{S}$ such that

$$
-\Delta \phi_{S}=s_{N}, \quad-\Delta \psi_{S}=s_{P} \quad \text { in } \Omega,
$$

still with Neumann and Dirichlet homogeneous boundary condition. Finally, one obtains the singular basis functions $\mathbf{x}_{S}$ of $\mathbf{X}_{S}$ by the aid of the relations

$$
\mathbf{v}_{S}=\operatorname{curl} \phi_{S}, \quad \mathbf{l}_{S}=\operatorname{grad} \psi_{S},
$$

together with relation (16). Hence, the keypoint is to compute $s_{N}$ or $s_{P}$. To this purpose, there exists several methods ([2], 8], etc.). We recall here briefly the method we choose, the Principal Part Method (see [9] for details). Consider, for simplicity reasons, a domain with one singularity. The Principal Part Method consists in splitting $s_{N}$ in a regular part $\tilde{s}_{N}$ (which belongs to $H^{1}(\Omega)$ ) and a known singular part $s_{N}^{P}$

$$
s_{N}=s_{N}^{P}+\tilde{s}_{N} .
$$

Above, $s_{N}^{P}$ belongs to $L^{2}(\Omega)$ but not to $H^{1}(\Omega)$, and verifies $\Delta s_{N}^{P}=0$. One thus computes, for instance by a $P^{1}$ finite element method, $\tilde{s}_{N}$ by solving

$$
\Delta \tilde{s}_{N}=0 \text { in } \Omega, \quad \frac{\partial \tilde{s}_{N}}{\partial \boldsymbol{\nu}}=-\frac{\partial s_{N}^{P}}{\partial \boldsymbol{\nu}} \text { on } \Gamma,
$$

avoiding thus mesh rafinement techniques.

One still uses singular mappings [6] to find the singular field $\phi_{S} \in H^{1}(\Omega) \cap$ $L_{0}^{2}(\Omega)$ such that

$$
-\Delta \phi_{S}=s_{N} \text { in } \Omega, \quad \frac{\partial \phi_{S}}{\partial \boldsymbol{\nu}}=0 \text { on } \Gamma
$$

Again, one can split this field in a regular part $\tilde{\phi}_{S}$ (which belongs to $H^{2}(\Omega)$ ) and a singular part $\phi_{S}^{P}$

$$
\phi_{S}=\tilde{\phi}_{S}+C_{\phi} \phi_{S}^{P}
$$

where $C_{\phi}$ is a constant to be determined. The principal parts $\phi_{S}^{P}$ is harmonic and does not belong to $H^{2}(\Omega)$. Its analytic expression is known. The regular part $\tilde{\phi}_{S}$ can be computed easily, by solving a standard variational formulation. The singular function $s_{P}$ and the singular field $\psi_{S}$ are computed in the same way.

Then, using a final time the singular mappings, one can compute the singular electromagnetic basis functions. To determine the basis $\mathbf{v}_{S}\left(\right.$ resp. $\mathbf{l}_{S}$ ), one simply takes the curl of $\phi_{S}$ (resp. the gradient of $\psi_{S}$ )

$$
\begin{aligned}
\mathbf{v}_{S} & =\operatorname{curl} \tilde{\phi}_{S}+C_{\phi} \operatorname{curl} \phi_{S}^{P}, \\
\mathbf{l}_{S} & =\nabla \tilde{\psi}_{S}+C_{\psi} \nabla \psi_{S}^{P},
\end{aligned}
$$


and $\mathbf{x}_{S}$ is easily obtained with (16). The singular constant $C_{\phi}$ can be computed by using a formula derived from integration by parts (cf. [9]). As an example, one gets

$$
C_{\phi}=\frac{\left\|s_{N}\right\|^{2}}{\int_{\Gamma} s_{N} \frac{\partial \phi_{S}^{P}}{\partial \boldsymbol{\nu}} d \Gamma}
$$

Now, we have to modify a classical method by handling the decomposition (13). We first write Ampère and Faraday's laws as two second-order in time equations. To enforce the divergence constraints on the electromagnetic field, we introduce two Lagrange multipliers (say $p$ for the electric field), to dualize Coulomb's and absence of free magnetic monopole's laws. In this way, one builds a mixed variational formulation ( $V F$ ) of Maxwell equations. For the electric field (the case of the magnetic field is handled similarly), this formulation reads Find $(\mathcal{E}, p) \in \mathbf{X} \times L^{2}(\Omega)$ such that

$$
\begin{aligned}
& \frac{d^{2}}{d t^{2}} \int_{\Omega} \mathcal{E} \cdot \mathbf{F} d \mathbf{x}+c^{2} \int_{\Omega} \operatorname{curl} \mathcal{E} \cdot \operatorname{curl} \mathbf{F} d \mathbf{x}+c^{2} \int_{\Omega} \operatorname{div} \mathcal{E} \operatorname{div} \mathbf{F} d \mathbf{x}+\int_{\Omega} p \operatorname{div} \mathcal{E} d \mathbf{x} \\
& \quad=-\frac{1}{\varepsilon_{0}} \frac{d}{d t} \int_{\Omega} \mathcal{J} \cdot \mathbf{F} d \mathbf{x}+\frac{c^{2}}{\varepsilon_{0}} \int_{\Omega} \rho \operatorname{div} \mathbf{F} d \mathbf{x}, \quad \forall \mathbf{F} \in \mathbf{X}, \\
& \int_{\Omega} \operatorname{div} \mathcal{E} q d \mathbf{x}=\frac{1}{\varepsilon_{0}} \int_{\Omega} \rho q d \mathbf{x} \quad \forall q \in L^{2}(\Omega) .
\end{aligned}
$$

To include the $S C M$ in this formulation, the electric field $\mathcal{E}$ is split like $\mathcal{E}(t)=$ $\mathcal{E}_{R}(t)+\mathcal{E}_{S}(t)$. The same splitting is used for the test functions of the variational formulation, which is discretized in time, with the help of the leap-frog scheme. From a practical point of view, we choose the Taylor-Hood, $P_{2}$-iso- $P_{1}$ Finite Element. In addition to being well-suited for discretizing saddle-point problems, it allows to build diagonal mass matrices, when suitable quadrature formulas are used. Thus, the solution to the linear system, which involves the mass matrix, is straightforward [1].

Then, one can write down the discrete singular part as a finite sum. Assuming again that there is one singularity, and let $\left(\mathbf{x}_{S}\right)$ be a given basis of the discrete singular space. One has $\mathcal{E}_{S}(t)=\kappa(t) \mathbf{x}_{S}$, where $\kappa$ is continous time-dependent function. This results in a fully discretized VF:

$$
\begin{array}{ll}
\mathbb{M}_{\Omega} \mathbf{E}_{R}^{n+1}+\mathbb{M}_{R S} \boldsymbol{\kappa}^{n+1}+\mathbb{L}_{\Omega} \mathbf{p}^{n+1} & =\mathbf{F}^{n}, \\
\mathbb{M}_{R S}^{T} \mathbf{E}_{R}^{n+1}+\mathbb{M}_{S} \boldsymbol{\kappa}^{n+1}+\mathbb{L}_{S} \mathbf{p}^{n+1} & =\mathbf{G}^{n}, \\
\mathbb{L}_{\Omega}^{T} \mathbf{E}_{R}^{n+1}+\mathbb{L}_{S}^{T} \boldsymbol{\kappa}^{n+1} & =\mathbf{H}^{n} .
\end{array}
$$

Above $\mathbb{M}_{\Omega}$ denotes the usual mass matrix, and $\mathbb{L}_{\Omega}$ corresponds to the divergence term involving $\mathbf{x}_{R}^{h}$ and the discrete Lagrange multiplier $p_{h}(t)$. Then, $\mathbb{M}_{R S}$ is a rectangular matrix, which is obtained by taking $\mathbf{L}^{2}$ scalar products between regular and singular basis functions, $\mathbb{M}_{S}$ is the "singular" mass matrix, and finally, $\mathbb{L}_{S}$ corresponds to the divergence term involving $\mathbf{x}_{S}$ and $p_{h}(t)$. To solve this system, one first removes the unknown $\boldsymbol{\kappa}^{n+1}$, so that the unknowns $\left(\mathbf{E}_{R}^{n+1}, \mathbf{p}^{n+1}\right)$ 
can be obtained with the help of a Uzawa-type algorithm. Finally, one concludes the time-stepping scheme by computing $\boldsymbol{\kappa}^{n+1}$ with the help of (26).

For error estimates for the singular functions $s_{N}, s_{P}$ and $\phi_{S}, \psi_{S}$, we refer the reader to [10]. As far as the electromagnetic sigular fields are concerned, error estimates are provided in [3. Let us briefly recall that, using the electric field splitting

$$
\mathcal{E}(t)=\mathcal{E}_{R}(t)+\mathcal{E}_{S}(t)=\mathcal{E}_{R}(t)+\kappa(t) \mathbf{x}_{S},
$$

one can prove, for a two-dimensional domain [11]

$$
\left|\kappa-\kappa_{h}\right| \leq C h^{2 \alpha},\left\|\mathcal{E}-\mathcal{E}_{h}\right\|_{\mathbf{X}} \leq C h^{2 \alpha-1-\varepsilon},\left\|\mathcal{E}-\mathcal{E}_{h}\right\|_{\mathbf{L}}^{2} \leq C h^{4 \alpha-2-\varepsilon},
$$

where $\pi / \alpha$ denotes here the reentrant angle of the non-convex domain $\Omega$.

\section{Numerical Experiments}

We consider an L-shaped domain $\Omega$, and assume its boundary is entirely perfectly conducting. Initial conditions are uniformly set to zero. A bunch of particles - electrons - is emitted from the top-most part of the boundary, with an initial velocity equal to $\mathbf{v}=v_{y} \boldsymbol{e}_{y}$, with $v_{y}=-2.10^{8} \mathrm{~ms}^{-1}$. The electromagnetic field is therefore a self-consistent field. Particles are absorbed at the down-most part of the boundary.
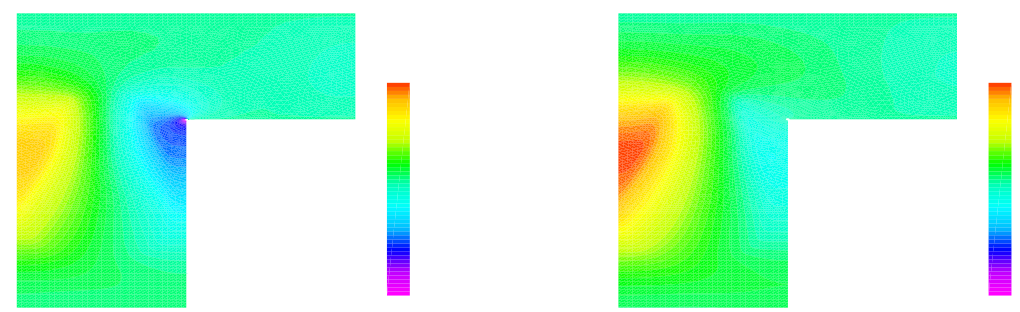

Fig. 1. electric field with (left) and without (right) SCM

As shown Figure 1 the electric field $-\mathcal{E}_{x}$ component - obtained after 500 timesteps is very different, when it is computed with and without the $S C M$. Recall that the electromagnetic field is the result of the motion of the charged particles only. Therefore, differences are entirely due to the coupling between the Vlasov and Maxwell equations.

\section{Conclusion}

In this paper, we proposed a new scheme for the time-dependent coupled VlasovMaxwell system of equations, in domains with singular geometries. The numerical experiments we have shown and their performance, make the study of such physical configurations possible. 


\section{References}

1. F. Assous, P. Degond, E. Heintzé, P. A. Raviart, J. Segré, On a finite element method for solving the three-dimensional Maxwell equations, J. Comput. Phys., 109, 222-237, 1993.

2. F. Assous, P. Ciarlet, Jr., J. Segré, Numerical solution to the time-dependent Maxwell equations in two-dimensional singular domain: The Singular Complement Method, J. Comput. Phys., 161, 218-249, 2000.

3. F. Assous, P. Ciarlet, Jr., E. Garcia, J. Segré, Time-dependent Maxwell's equations with charges in singular geometries, submitted to Comput. Methods Appl. Mech. Engrg..

4. P.A. Raviart, An Analysis of Particle Methods, Springer-Verlag, Berlin, 1985.

5. F. Assous, P. Degond, J. Segré, A particle-tracking method for 3D electromagnetic PIC codes on unstructured meshes, Comput. Phys. Com., 72, 105-114, 1992.

6. E. Garcia, Résolution des équations de Maxwell avec charges dans des domaines non convexes, PhD Thesis, University Paris 6, France, 2002. (in French)

7. P. Grisvard, Singularities in boundary value problems, 22, RMA Masson, Paris, 1992.

8. C. Hazard, S. Lohrengel A singular field method for Maxwell's equations: numerical aspects for 2D magnetostatics, SIAM J. Appl. Math., 40, 1021-1040, 2002.

9. F. Assous, P. Ciarlet, Jr., S. Labrunie, J. Segré, Numerical solution to the timedependent Maxwell equations in axisymmetric singular domain: The Singular Complement Method, J. Comput. Phys., 191, 147-176, 2003.

10. P. Ciarlet, Jr.,J. He, The singular complement method for $2 \mathrm{D}$ scalar problems, $C$. R. Acad. Sci. Paris, Ser. I, 336, 809-814 (2005).

11. E. Jamelot, A nodal finite element method for Maxwell's equations, C. R. Acad. Sci. Paris, Ser. I, 339, 353-358 (2003). 